\section{Comparison of ICG- assisted ILM peeling and triamcinolone- assisted posterior vitreous removal in diffuse diabetic macular oedema}

\begin{abstract}
Aim To compare the effect of indocyanine green (ICG)-assisted internal limiting membrane (ILM) peeling and triamcinolone acetonide-assisted posterior vitreous removal on visual acuity in patients with diffuse diabetic macular oedema (DMO).

Methods In total, 24 patients with diffuse DMO who underwent pars plana vitrectomy were included in this study. In all, 11 patients (mean age 57 years) were performed ICGassisted ILM peeling; while 13 patients (mean age 54 years) underwent triamcinoloneassisted posterior vitreous removal. Patients from two different treatment regimens were compared in terms of best-corrected visual acuity (BCVA) at postoperative sixth months. Results In ICG-assisted ILM peeling group, preoperative BCVA $(1.3 \pm 0.4$, mean $\pm S D$, $\log$ MAR) improved postoperatively to $0.9 \pm 0.5$ $(P=0.011)$. In eyes underwent triamcinoloneassisted posterior vitreous removal, baseline BCVA of $1.4 \pm 0.4$ improved to $1.0 \pm 0.5$ $(P=0.007)$. There was no difference between baseline as well as postoperative sixth-month BCVA results of both groups $(P=0.59$ and $P=0.57$, respectively).

Conclusions Triamcinolone-assisted posterior vitreous removal and ICG-assisted ILM peeling have the same effect in terms of postoperative BCVA in patients with diffuse DMO.
\end{abstract}

Eye (2006) 20, 1357-1359. doi:10.1038/sj.eye.6702152; published online 18 November 2005

Keywords: diffuse diabetic macular oedema; indocyanine green; internal limiting membrane; posterior vitreous; triamcinolone

Y Bardak, O Çekiç and ŞU Tiğ

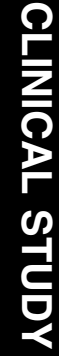

\section{Introduction}

Diabetic Macular Oedema (DMO) is common cause of visual loss in diabetics. The treatment is based mainly on laser photocoagulation but results are unsatisfactory. ${ }^{1}$ Intravitreal triamcinolone injection have been successfully tried for DMO. However, the need for reinjections and various complications are the main obstacles. ${ }^{2}$

The role of vitreoretinal traction in the pathogenesis of DMO as well as positive impact of vitrectomy and removal of internal limiting membrane (ILM) on DMO has been known. ${ }^{3-6}$ Triamcinolone-assisted posterior vitreous separation is also beneficial for DMO.,

We compared the efficacy of indocyanine green (ICG)-assisted ILM peeling and triamcinolone-assisted posterior vitreous separation in terms of best-corrected visual acuity (BCVA) in DMO.

\section{Materials and methods}

In total, 24 type II Diabetes Mellitus patients with diffuse DMO who underwent pars plana vitrectomy were evaluated retrospectively. Signed informed consent was obtained from the patients. The patients were randomly assigned into two groups: 11 patients ( 5 female) with a mean age of 57 years (range 48-64) underwent ICG-assisted ILM peeling; while 13 patients (6 female) with a mean age of 54 years (range, 47-61) underwent triamcinolone-assisted posterior vitreous removal. Diffuse DMO was the main surgical indication for all patients that had been suffering from significant loss of vision within last 6 months. The diagnosis of diffuse DMO mostly based on clinical
Department of

Ophthalmology, Süleyman Demirel University Medical School, Isparta, Turkey

Correspondence:

Y Bardak, Department of Ophthalmology, Suleyman Demirel University, Medical School, Isparta, 32260

Turkey

Tel: + 90246211 2308;

Fax: +902462181347.

E-mail: yavuzbardak@

hotmail.com

Received: 1 June 2005 Accepted in revised form: 17 September 2005

Published online:

18 November 2005

Financial Interest: No

This study was partly presented at Fourth European Vitreoretinal Society Meeting, Istanbul, Turkey, September 2004 and was selected as the second best poster 
examination. Fundus photographs and fluorescein angiography were obtained when needed. At least 3 months before the operation, patients had laser treatment, failed to achieve fluid absorption, and additional laser treatment was not advised. Patients with tractional macular detachment, dense vitreous haemorrhage, and posterior vitreous detachment were excluded. Preoperative and postoperative 6-month BCVA examination results were analysed.

During operation, either $0.1 \mathrm{ml}$ of ICG $0.25 \%$ or $0.5-1.0 \mathrm{ml}$ of triamcinolone acetonide $(40 \mathrm{mg})$ was used as a surgical adjunct. Intraoperative scatter laser photocoagulation was performed in all eyes.

Pre- and postoperative 6-month logMAR BCVA values were compared with Mann-Whitney and Wilcoxon tests.

\section{Results}

Figure 1 shows the pre- and postoperative 6 months BCVA in groups. In ILM peeling group, preoperative BCVA (1.3 \pm 0.4 , mean \pm SD, logMAR; Snellen fraction: $0.05)$ improved to $0.9 \pm 0.5$ (Snellen fraction: 0.125 ) $(P=0.011)$. In eyes underwent triamcinolone-assisted posterior vitreous removal, baseline BCVA of $1.4 \pm 0.4$ (Snellen fraction: 0.04) improved to $1.0 \pm 0.5$ (Snellen fraction: 0.1$)(P=0.007)$. There was no difference in baseline as well as postoperative 6 months BCVA results of both groups ( $P=0.59$ and $P=0.57$, respectively). At the last follow-up, there was an improvement in DMO of all cases based on clinical and/or fundus florescein angiographic exams.

\section{Discussion}

Prospective studies reported $43 \%^{6}$ and $52 \%{ }^{5}$ of eyes with DMO gained two or more lines of visual acuity following ILM peeling. Performing vitrectomy without ILM peeling in eyes with no evident vitreomacular traction, LaHeij et $a l^{3}$ reported resolution of DMO in 21 of 21 eyes while Tachi and Ogino ${ }^{4}$ reported resolution of DMO in 57 of 58 eyes. These favourable results make ILM removal be questionable. ICG staining facilitates and accelerates ILM peeling but a potential toxic effect of ICG should be kept in mind. ${ }^{9}$ Haritoglou et $a l^{10}$ detected visual field defects after ICG staining during vitrectomy. By removing ILM, we can be sure of releasing tractional forces on the macula. As ILM plays an important role as a scaffold for proliferating astrocytes, the removal of the ILM may prevent the development of epiretinal membranes after vitrectomy for DMO. ${ }^{11}$

A certain number of eyes with DMO did not have visual recovery even after a successful vitrectomy. ${ }^{7}$ Diabetic eyes had a greater chance of having a residual vitreous cortex after posterior vitreous removal, as demonstrated by the
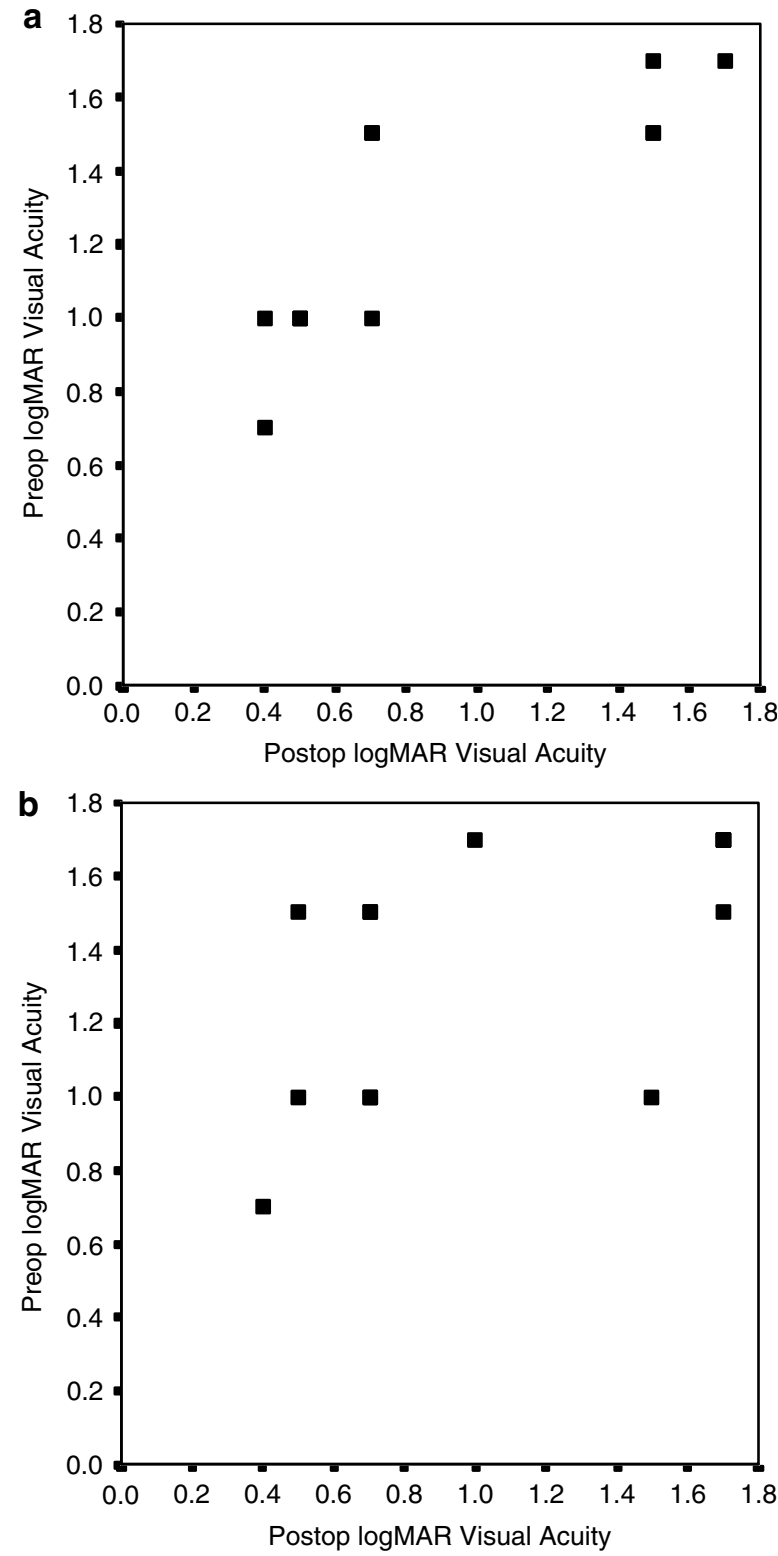

Figure 1 (a) Pre- and postoperative BCVA (logMAR) in ICGassisted ILM peeling group. (b) Pre- and postoperative BCVA in triamcinolone-assisted posterior vitreous removal group.

triamcinalone-assisted vitrectomy. ${ }^{6}$ Failure to recognise true posterior vitreous hyaloid may contribute to continued morbidity after vitrectomy. Residual vitreous on the retina left untreated during vitrectomy may serve as a scaffold for continued neovascular proliferation and release inflammatory mediators leading to complications of proliferative diabetic retinopathy. ${ }^{6}$ The triamcinoloneassisted vitrectomy provides better removal of the posterior vitreous cortex. ${ }^{7}$

We believe that ILM peeling provides complete removal of macular tractions and vitreous remnants after surgical posterior vitreous separation acting as an 
inflammatory focus. Therefore, if triamcinolone-assisted posterior vitreous separation is performed, then ILM removal may not be needed. This study showed that, triamcinolone-assisted posterior vitreous removal and ICG-assisted ILM peeling have the same effect on BCVA in $\mathrm{DMO}$, but further studies with large number of cases and longer follow-up time may better clarify the efficacy of these techniques for functional outcome.

\section{References}

1 Early Treatment Diabetic Retinopathy Study Research Group. Photocoagulation for diabetic macular edema. ETDRS report no 1. Arch Ophthalmol 1985; 103: 1796-1806.

2 Çekiç O, Chang S, Tseng JJ, Akar Y, Barile GR, Schiff WM. Cataract progression after triamcinolone injection. Am J Ophthalmol 2005; 139: 993-998.

3 La Heij EC, Hendrikse F, Kessels AGH, Derhaag PJFM. Vitrectomy results in diabetic macular oedema without evident vitreomacular traction. Graefe's Arch Clin Exp Ophthalmol 2001; 239: 264-270.

4 Tachi N, Ogino N. Vitrectomy for diffuse macular edema in cases of diabetic retinopathy. Am J Ophthalmol 1996; 122: 258-260.

5 Avc1 R, Kaderli B, Avcı B, Simsek S, Baykara M, Kahveci Z et al. Pars plana vitrectomy and removal of the internal limiting membrane in the treatment of chronic macular oedema. Graefe's Arch Clin Exp Ophthalmol 2004; 242: 845-852.

6 Dillinger P, Mester U. Vitrectomy with removal of the internal limiting membrane in chronic diabetic macular oedema. Graefe's Arch Clin Exp Ophthalmol 2004; 242: 630-637.

7 Peyman GA, Cheema R, Conway MD, Fang T. Triamcinolone acetonide as an aid to visualization of the vitreous and the posterior hyaloid during pars plana vitrectomy. Retina 2000; 20: 554-555.

8 Sonoda KH, Sakamoto T, Enaida H, Miyazaki M, Noda Y, Nakamura T et al. Residual vitreous cortex after surgical posterior vitreous separation visualized by intravitreous triamcinolone acetonide. Ophthalmology 2004; 111: 226-230.

9 Çekiç O, Morimoto T, Ohji M, Sawa M, Hasegawa T, Sakaguchi $\mathrm{H}$ et al. Nonaxoplasmic transfer of indocyanine green into the optic nerve after intravitreal application. Retina 2004; 24: 412-415.

10 Haritoglou C, Gandorfer A, Gass CA, Schaumberger M, Ulbig MW, Kampik A. The effect of indocyanine-green on functional outcome of macular pucker surgery. Am J Ophthalmol 2003; 135: 328-337.

11 Pendergast SD, Hassan TS, Williams GA, Cox MS, Margherio RR, Ferrone PJ et al. Vitrectomy for diffuse diabetic macular edema associated with a taut premacular posterior hyaloid. Am J Ophthalmol 2000; 130: 178-186. 